Cahiers de recherches médiévales

\title{
La figure de Jules César dans le Discours de la servitude volontaire de La Boétie et dans les Essais de Montaigne
}

Bénédicte Boudou et Françoise Charpentier

\section{OpenEdition}

\section{Édition électronique}

URL : https://journals.openedition.org/crm/858

DOI : $10.4000 / \mathrm{crm} .858$

ISSN : 1955-2424

\section{Éditeur}

Honoré Champion

Édition imprimée

Date de publication : 30 mars 2006

Pagination : 183-203

ISSN : 1272-9752

Référence électronique

Bénédicte Boudou et Françoise Charpentier, « La figure de Jules César dans le Discours de la servitude volontaire de La Boétie et dans les Essais de Montaigne», Cahiers de recherches médiévales [En ligne], 13 spécial | 2006, mis en ligne le 03 avril 2009, consulté le 15 décembre 2022. URL : http:// journals.openedition.org/crm/858; DOI : https://doi.org/10.4000/crm.858 


\section{RM}

\section{La figure de Jules César \\ dans le Discours de la servitude volontaire de La Boétie et dans les Essais de Montaigne ${ }^{1}$}

\section{La figure de Jules César dans les Essais}

Dans les débats idéologiques en Italie ${ }^{2}$, la figure de Jules César se situe au cœur de la grande polémique entamée par les humanistes florentins contre leurs homologues milanais et contre les visées expansionnistes de la tyrannie incarnée par les Visconti, et César est un des personnages les plus cités chez Machiavel. Même fascination en France : Marot se réfère à Jules César pour célébrer le vainqueur de Cérisoles $^{3}$, dans le Liber de militia, Pierre Ramus cherche, à partir des actions de César, à établir les règles d'action du général en chef $(1559)^{4}$, tandis que c'est plutôt la mort de César qui retient les auteurs de tragédies. Les pièces de Marc-Antoine Muret (Montaigne semble avoir joué dans son Julius Casar ${ }^{5}$ ) et de Jacques Grévin proposent une réflexion politique et s'interrogent sur ce qui a suivi l'assassinat de César : doit-on se réjouir de la mort du tyran, ou craindre un redoublement de violence et l'effondrement de la puissance romaine ? Avec celle de Shakespeare (1599), ces tragédies donnent à lire l'ambivalence du personnage de César aux yeux des humanistes. Elles les montrent aussi appliqués à déchiffrer les événements romains à la lumière des guerres civiles en France, et elles posent la question de l'utilité d'un tyrannicide qui n'a pas libéré Rome de la tyrannie. On retrouve la même problématique politique dans les Vindicia contra tyrannos ${ }^{6}$ évoquant, entre autres tyrannies, celle de César «...qui avoit envahi l'Estat par violence, neantmoins pour endormir le

\footnotetext{
${ }^{1}$ Les auteurs de ce travail se sont mis d'accord sur l'ensemble du texte, y compris sur ses divergences. La première partie, «La figure de Jules César dans les Essais », est due à Bénédicte Boudou. La seconde, «La pensée politique sur Jules César, de Montaigne à La Boétie », à Françoise Charpentier.

${ }^{2}$ R. Baillet, «César chez Machiavel », Présence de César, Hommage au doyen M. Rambaud, Actes du colloque de décembre 1983, Paris, Belles Lettres, 1985, p. 67-76, ici p. 67.

${ }^{3}$ Vers 11 à 13 de l'Epistre à M. d'Anguyen : «Ainsi ayant ce que Cesar avoit/ Qui est celluy qui à l'œil bien ne voit/ Qu'impossible est qu'en armes ne l'imite », Euvres complètes, éd. G. Defaux, 1993, t. 2, p. 707. Voir J. Pineaux, «César dans la tragédie humaniste de la Renaissance française », Présence de César, op. cit., p. 213

${ }^{4}$ Voir Cl.-G. Dubois, «César et Ramus », Présence de César, p. 112

${ }^{5}$ Les références aux Essais seront données successivement dans l'édition Villey-Saulnier, PUF, 1965 (VS), et dans l'édition de la Pochothèque, Hachette, 2001 (P). Des chapitres 14 à $41 \mathrm{du}$ livre I, un décalage d'un chapitre existe entre les deux éditions, si bien que le chapitre sera précisé pour chacune des deux éditions. Sinon, seule la référence de page différenciera les deux éditions. Essais, VS. I, 26, 176B/P. I, 25, 265 : «J'ai soutenu les premiers personnages ès tragédies latines de Buchanan, de Guerente et de Muret, qui se représentèrent en notre collège de Guyenne ».

${ }^{6} \mathrm{Ou}$ Revendications contre les tyrans, traitant De la puissance légitime du prince sur le peuple et du peuple sur le prince, d'Eugène Junius Brutus, 1581.
}

Cahiers de Recherches Médiévales, 13spé, 2006 
monde sous quelque couleur d'equité, [et qui] vouloit que l'on creust qu'il avoit esté establi Empereur par le peuple et par le senat» ${ }^{7}$. De son côté, Simon Goulart «augmente» les Vies de Plutarque d'une Comparaison d'Alexandre le grand avec Julius Casar ${ }^{8}$ et, tout en jugeant César militairement supérieur à Alexandre, il célèbre la vertu royale d'Alexandre tandis qu'il dénonce César qui, «faisant du liberal et du harangueur pour briguer les uns et les autres», «viola la liberté de Rome»".

Les Essais dans lesquels on a vu, du moins pour les deux premiers livres, des discours militaires, font également une large place à Jules César ${ }^{10}$. $\mathrm{M}^{\mathrm{lle}}$ de Gournay agit ici en lectrice-témoin puisque, dès la première phrase de sa préface à l'édition de 1595, elle le mentionne : «Si vous demandez à quelque artisan, quel est Cæsar, il vous répondra que c'est un excellent Capitaine $»^{11}$. Dans les Essais, César est présent à plus d'un titre : il est d'abord admiré comme chef d'armée, qualifié avec Alexandre de «miracle en l'art militaire $»^{12}$ ou de «plus grand capitaine du monde $»^{13}$. C'est à cette figure de César qu'est consacré le chapitre 34 du livre II. Mais César est encore reconnu comme orateur et écrivain : le chapitre «Des Livres» le met audessus de «tous les historiens » et de «Cicero même $»^{14}$. Enfin, il est présenté comme une personnalité à multiples facettes qui suscite les interrogations de Montaigne et stimule son discernement.

Je m'interrogerai d'abord sur les sources auxquelles puise Montaigne pour dessiner la figure de Jules César, ce qui permettra de déterminer quel César il fait apparaitre dans les Essais. Je me demanderai ensuite si l'on peut conclure à une disparition progressive de César chez Montaigne ou si, plutôt, on est confronté à une coexistence d'affirmations contradictoires concernant le personnage.

Un «miracle de l'art militaire »

Les sources auxquelles Montaigne puise pour chercher à cerner le personnage sont d'abord les Commentaires de César, sur lesquels il porte 681 annotations. Cette abondance ne rend pas compte de l'utilisation ténue qu'il en fait dans les Essais. Catherine Séguier-Leblanc ${ }^{15}$ a confronté au texte des Essais les nombreuses annotations que Montaigne écrit dans les années 1578 sur ses exemplaires de la Guerre des

\footnotetext{
${ }^{7}$ P. 102 .

${ }^{8}$ Vies des hommes illustres reveuës, corrigees et augmentees en ceste derniere edition de plusieurs comparaisons de vies..., Paris, Cl. Morel, 1619, tome 2, fo 481-485.

${ }^{9}$ Il se souvient de Montaigne dont il recopie presque littéralement certaines phrases du chapitre II, 34: «Et cependant on le trouvera beaucoup plus retenu en ses entreprises qu'Alexandre, qui court à force les dangiers comme un torrent impetueux, lequel choque indifferemment tout ce qu'il rencontre : aussi estoit-il en la chaleur de son aage, et Cæsar entra en affaire estant desja meur et advancé », op. cit., $\mathrm{f}^{\circ} 484$.

${ }_{10}$ Notons les quelques études déjà faites sur César chez Montaigne: R. Chevalier, «Montaigne lecteur et juge de César», Présence de César, Casarodunum XX bis, 1985, p. 91-105, et J.-M. Compain, «Montaigne, admirateur de César? », Montaigne et l'histoire, Actes du colloque de Bordeaux 1988, Paris, Klincksieck, 1991, p. 67-72.

${ }^{11}$ Éd. J. Céard et al., Pochothèque, p. 10.

${ }^{12}$ I, 48, VS. 288/P. 470.

${ }^{13}$ II, 17, VS. 632/P. 975.

${ }^{14}$ II, 10, VS. 416/ P. 659.

${ }^{15}$ Montaigne Studies, « Montaigne et les anciens », 2005, p. 177.
} 
Gaules et de la Guerre Civile. Avant de conclure à une lente digestion de la Guerre Civile dans les Essais, elle mentionne par exemple que les dix-neuf notes de Montaigne concernant les exploits de Labienus en Gaule se réduisent à deux allusions explicites au chapitre II, $8^{16}$ et en II, $32^{17}$, où il est question de "capitaines étouffés sous la splendeur d'autres noms, de moindre mérite». Montaigne s'est souvent appliqué à montrer que des hommes d'action sont restés obscurs et ont renoncé à la gloire afin de sauver celle de leurs maîtres : c'est encore le sujet du chapitre intitulé «Des récompenses d'honneur», I, 41.

Les autres sources de Montaigne pour Jules César sont Dion Cassius, Plutarque, et Suétone. Il semble que Montaigne n'ait pas lu directement Dion Cassius ${ }^{18}$ et qu'il soit passé par le biais de la Methodus de Jean Bodin. Or, alors que Dion donne l'avantage moral à Pompée ${ }^{19}$, Montaigne retient de lui l'admiration qu'il voue à César. De Plutarque, Montaigne a gardé l'armature d'une vie, une armature qui s'attache surtout, après la description des débuts de César et du premier triumvirat, à la guerre des Gaules. L'auteur des Essais écarte le début de la vie de César chez Plutarque : il passe sous silence l'animosité, mise en valeur dès le chapitre IV, de Cicéron «qui connut la rusée malice qu'il cachait sous le manteau de [sa] privauté ». Il néglige le chapitre VI qui souligne que César achète la faveur publique, dont La Boétie se souviendra particulièrement. Il s'inspire de Plutarque surtout à partir du chapitre XIX qui marque un changement de ton, Plutarque se mettant à louer le grand capitaine que fut César et sa vertu militaire. Mais si l'auteur des Vies fait une place à la rivalité entre César et Pompée et à la guerre civile, il passe rapidement sur la fin de la vie de César, c'est-à-dire sur l'homme d'État qui, consul à vie, soumet la République romaine à une dictature, gouverne en souverain absolu, réduit le pouvoir du sénat, relève Carthage et Corinthe et rêve de devenir roi. La fin de la vie de César (que Plutarque a transférée dans la Vie de Brutus) est également absente chez Montaigne, qui condamne à plusieurs reprises sa «pestilente ambition». La lecture de Montaigne n'est pas tout à fait fidèle à Plutarque : Plutarque célébrait bien sûr la vertu militaire du personnage, mais il soulignait ses hésitations, avant le passage du

\footnotetext{
${ }^{16}$ VS. 400/P. 634.

${ }^{17}$ VS. 726/P. 1130.

${ }^{18}$ Histoire romaine, Livres 40-41, Paris, Belles Lettres, La roue à livres, introd., trad. et notes par Michèle Rosellini, 1996. Dion insère le récit de la guerre des Gaules à l'intérieur du récit de la guerre civile, et s'attache particulièrement à la rivalité entre Pompée et César. Il montre Pompée hésitant et mal préparé, agissant contrairement à ce qu'on exige d'un chef militaire et d'un homme d'État. XLI, 4, 2 : «Pompée prit peur»; XLI, 13, 1 «Pompée quitta donc ainsi sa patrie »; XL, 13, 4 «c'est chargé de l'humiliation que lui valait sa peur de César qu'il repartit, et, alors qu'il était fameux pour avoir accru la puissance de sa patrie, il était devenu, par sa désertion, la plus infâme qui fût». Il souligne la supériorité tactique de César, qui a pour lui la rapidité de décision et d'intervention XLI, 44, 1: «César saisit l'occasion de la guerre », sait agir sur ses hommes, est habile à redresser une situation et à se tirer du désarroi, en particulier à Dyrrachium.

${ }^{19}$ XLI, 54, 1 : «tandis que l'un [Pompée] était soucieux d'être honoré par des gens consentants, obéi avec leur assentiment et aimé, l'autre ne se faisait pas scrupule de gouverner le peuple même contre son gré, de donner des ordres à des hommes qui le détestaient, et de s'attribuer les honneurs de sa propre autorité ».
} 
Rubicon (chapitre XLII) et avant de s'embarquer pour Brindisi (chapitre XLIX) ${ }^{20}$. Montaigne ne partage apparemment pas cette vision du personnage, il dessine même César en face de l'expédition d'Égypte comme quelqu'un qui veut «exécuter, non pas consulter les plus hautes entreprises $»^{21}$. Mais il retient, en l'épurant ${ }^{22}$, l'image des pleurs de César à qui l'on présente la tête de Pompée assassinéén ${ }^{23}$ que Plutarque n'interprète pas comme un signe d'hypocrisie, ce que faisait Lucain ${ }^{24}$. Et il donne les attendus d'un jugement qui milite pour la sincérité de César:

Il y avait eu entre eux une si longue intelligence et société au maniement des affaires publiques, tant de communauté de fortunes, tant d'offices réciproques et d'alliance, qu'il ne faut pas croire que cette contenance fut toute fausse et contrefaite, comme estime cet autre $[\ldots]^{25}$.

Une fois n'est pas coutume, moins que de Plutarque, c'est surtout de Suétone, exceptionnellement digne dans le récit qu'il donne de la vie de César, que Montaigne s'inspire. Mais il lit Suétone à la manière de Plutarque, c'est-à-dire qu'il s'intéresse moins à l'homme public décrit par les quarante-quatre premiers chapitres du César de Suétone, qu'à l'homme privée ${ }^{26}$ présenté dans les trente derniers ${ }^{27}$.

À partir de ces sources, Montaigne ébauche un portrait qui, s'il est un peu composite, privilégie en Jules César le chef de guerre tel que le présentent Suétone et les Commentaires de César lui-même dont la «riche matière» est «fardée [...] d'une façon de dire si pure, si délicate et si parfaite $»^{28}$. C'est tout à fait caractéristique au chapitre «De la Présomption », qui rappelle la nécessité de savoir se reconnaître tel qu'on est et illustre cette idée par l'exemple de César ${ }^{29}$ : «Si c'est César, qu'il se trouve hardiment le plus grand capitaine du monde $»^{30}$. César est ce chef de guerre qui «opposait seul l'autorité de son visage et la fierté de ses paroles »" à ses soldats, et qui «marchait toujours devant sa troupe $»^{32}$. De façon tout à fait explicite, le chapitre 34 du livre II ( Observations sur les moyens de faire la guerre de Jules Cæsar ») se consacre à «ce patron de l'art militaire »: la première partie du chapitre

\footnotetext{
${ }^{20}$ "Ne sachant ce qu'il devait faire », écrit Plutarque.

${ }^{21}$ II, 34, VS. 740/P. 1151.

${ }^{22}$ César chez Montaigne détourne la tête. Chez Plutarque, il pleure en prenant le cachet de Pompée.

${ }^{23}$ Chapitre LXII. Essais, VS. I, 38, 233/P. I, 37, 361 : «Quand on présenta à Cæsar la tête de Pompée, les histoires disent qu'il en détourna sa vue, comme d'un vilain et mal plaisant spectacle. »

${ }^{24}$ «... Il répand des larmes contraintes, et fait sortir des plaintes de son cœur satisfait », Pharsale, liv. IX, v. 1042.

${ }^{25}$ VS. I, 38, 233/P. I, 37, 361.

${ }^{26}$ Plus exactement, il puise dans les chapitres $45,48,50$ à 53,56 à 58,65 à 67,72 à 75 .

${ }^{27}$ Chapitres 45 à 75 . A noter : Montaigne néglige les deux derniers chapitres de Suétone dont le dernier citait des mots de César.

${ }^{28}$ II, 34, VS. 736 A/ P. 1144.

${ }^{29}$ Idée reprise par la phrase liminaire de la préface de $\mathrm{M}^{\text {lle }}$ de Gournay.

${ }^{30} \mathrm{II}, 17$, VS. 732/ P. 975.

${ }^{31}$ VS. I, 24, 130/ P. I, 23, 199.

${ }^{32}$ VS. I, 36, 226/P. I, 35, 351.
} 
s'arrête sur quelques «traits particuliers et rares » puisés dans les Commentaires de César, dont Montaigne offre un véritable florilège, tandis que la seconde partie ébauche un parallèle entre César et Alexandre. Attardons-nous ici sur quelques-uns de ces traits. César est habile à pratiquer l'art de la surprise et à "prendre au point » les occasions ${ }^{33}$. Il sait intervenir rapidement : «la diligence, qui est en ses exploits à la vérité inouïe et incroyable $\aleph^{34}$. Il témoigne d'une grande autoritée ${ }^{35}$, comme l'attestent l'attitude de ses soldats à Dyrrachium, mais aussi leur fidélité et leur courage; il n'exige d'eux que la vaillance, les appelle ses compagnons et les pare pour les combats ${ }^{36}$. Il a l'art de les exhorter par les «grâces particulières » en son parler, comme le montre la bataille de Tournai ${ }^{37}$. Malgré cette autorité, César fait beaucoup de choses lui-même, qu'il s'agisse de reconnaître les lieux ou de sonder les gués: «Cæsar, dit Suetone ${ }^{38}$, marchait toujours devant sa troupe, à pied, la tête découverte $»^{39}$. Montaigne cite encore un autre trait qui l'a frappé chez César parce qu'il va à l'encontre de ce que recommanderait l'habileté : dans la guerre contre le roi Juba, au lieu de minimiser les forces de l'ennemi pour encourager ses soldats, César les grossit ${ }^{40}$. Quant aux entreprises comme celle d'Égypte ou la bataille d'Alésia, elles «surpassent en hasard tout discours de raison militaire ${ }^{41}$. Vaillant capitaine, César est respectueux de ses ennemis :

La voie qu'y tint J. Cæsar, je trouve que c'est la plus belle qu'on puisse prendre. Premièrement il essaya par clémence et douceur à se faire aimer de ses ennemis mêmes ${ }^{42}$.

Mais si l'homme de guerre est au premier plan, Montaigne aime également en César l'historien qui a su allier le faire au dire, plus exactement celui dont «les gestes», comme celles de Xénophon, ont «de bien loin surpassé l'éloquence ${ }^{43}$. C'est d'ailleurs par cette éloquence que Montaigne explique l'ascendant qu'a pris César $^{44}$. Il apprécie son style «soldatesque » qui l'oppose à Cicéron ${ }^{45}$ et l'estime

mériter qu'on l'étudie, non pour la science de l'histoire seulement, mais pour luimême, tant il a de perfection et d'excellence par-dessus tous les autres ${ }^{46}$.

\footnotetext{
${ }^{33}$ VS. 736/ P. 1145.

${ }^{34}$ VS. 737, P. 1145.

${ }^{35}$ «Jamais chef de guerre n'eut tant de créance sur ses soldats», II, 34, VS. 742/P.1155, ou encore «Il accoutumait surtout ses soldats à obéir simplement », II, 34, VS. 736/P. 1145.

${ }^{36}$ Le fait est mentionné deux fois.

${ }^{37}$ VS. 738./ P. 1148.

${ }^{38}$ Chapitre 57.

${ }^{39}$ VS. I, 36, 226/P. I, 35, 351.

${ }^{40}$ II, 34, VS. 736/P. 1144-1145.

${ }^{41}$ VS. 740/ P. 1151.

${ }^{42}$ VS. I, 24, 131/P. I, 23, 201. De même au chapitre «De la cruauté » «Il était doux en ses vengeances », II, 11, VS. 430/P. 680.

${ }^{43}$ VS. I, 40, 249/P. I, 39, 386.

${ }^{44}$ I, 51, VS. 305/P. 496.

${ }^{45}$ VS. I, 26, 172/P. I, 25, 265.

${ }^{46}$ II, 10, VS. 416/P. 659.
} 
Un court chapitre des Essais donne voix à « un mot de Cæsar» :

Il se fait $[\ldots]$ que nous ayons et plus de fiance et plus de crainte des choses que nous n'avons pas vues, et qui sont cachées et inconnues ${ }^{47}$,

et quelques autres passages font entendre un César penseur qui a marqué Montaigne: «J'ai essayé en plusieurs occurrences ce que dit Cæsar, que les choses nous paraissent souvent plus grandes de loin que de près $»^{48}$, ou encore : «Cæsar tient qu'il est plus souvent advenu que la renommée a devancé l'accident $»^{49}$. Par deux fois ${ }^{50}$ enfin, Montaigne cite le mot de César sur la meilleure mort : «la moins préméditée et la plus courte».

Pourtant, le portrait que les Essais brossent de César est largement un portrait en miettes, en éclats brisés, pour tout dire composite. Lecteur assidu de Plutarque, c'est l'homme que Montaigne cherche à saisir derrière l'image officielle d'un personnage, cette image fût-elle même celle que le personnage a livrée de lui. Et il s'émerveille des multiples facettes de César parce qu'il voit en lui en quelque sorte un échantillon de l'humaine nature :

Tout mouvement nous découvre. Cette même âme de Cæsar, qui se fait voir à ordonner et dresser la bataille de Pharsale, elle se fait aussi voir à dresser des parties oisives ou amoureuses ${ }^{51}$.

Dans l' «Histoire de Spurina», Montaigne parle même de «disparité des passions » chez César, en qui coexistèrent l'amour et l'ambition.

À cette disparité du personnage de César tel que Montaigne le représente s'ajoute l'irrégularité de ses apparitions dans les Essais, qui ont parfois fait conclure à une disparition progressive de César.

Une disparition de César?

Quand on observe la distribution des références à Jules César dans les Essais, on se rend compte que sur 115 occurrences, César est présent plus d'une quarantaine de fois dans le livre I (43 fois dans vingt chapitres), cinquante-huit fois dans le livre II (dans dix-huit chapitres, dont deux consacrés à César, chapitres 33 et 34), tandis qu'il n'apparaît que quatorze fois dans le livre III $^{52}$. Plus significatif, dans les premiers livres, César est mentionné soit seul, soit en présence d'hommes auxquels il

\footnotetext{
${ }^{47}$ I, 53, VS. 310/P. 503.

${ }^{48}$ VS., I, 20, 90/P. I, 19, 137.

${ }^{49}$ VS. I, 27, 180/ P. I, 26, 279.

${ }^{50}$ II, 13, VS. 608/P. 938, et III, 12, VS. 1052/P. 1634 : «l'opinion de Cæsar que la moins pourpensée mort était la plus heureuse ».

${ }_{51}$ I, 50, VS. 302/P. 490-491

${ }_{52}$ Aux chapitres 4, 5, 6, 9, 10, 12 et 13 (mais César est évoqué sans être nommé aux chapitres III, 1 et III, 7).
} 
est égalé, qu'il s'agisse de Pompée ${ }^{53}$, de Xénophon ${ }^{54}$, ou d'Alexandre ${ }^{55}$. Et il n'est évoqué qu'une seule fois de façon injurieuse, au chapitre «De la cruauté ». Montaigne y parle de Caton et écrit :

Je tomberais aisément en cette opinion, qu'il savait bon gré à la fortune d'avoir mis sa vertu à une si rude épreuve, et d'avoir favorisé ce brigand ${ }^{56}$ à fouler aux pieds l'ancienne liberté de sa patrie ${ }^{57}$.

Dans le troisième livre en revanche, César n'est évoqué seul qu'à deux reprises : au chapitre III, 13, pour sa résistance à l'épilepsie ${ }^{58}$, et (un peu plus haut) pour se voir ôter tout privilège particulier : «la vie de Cæsar n'a point plus d'exemple que la nôtre pour nous $»^{59}$. Le reste du temps, César est accouplé à un autre personnage historique, qui est souvent Alexandre ${ }^{60}$. Et s'il est encore associé à Pompée, c'est pour lui être égalé ou même pour être rejeté : «Entre Cæsar et Pompee je me fusse franchement declaré ${ }^{61}$. Sinon, César n'est pas même nommé ; il n'a droit qu'à une allusion insultante. C'est le cas à la fin du chapitre III, 1 , où Montaigne, après avoir cité des vers de Lucain peignant l'avidité guerrière du vainqueur des Gaules ${ }^{62}$, commente : «j'abomine les enhortements enragés de cette autre âme déréglée » ${ }^{63}$. Tel est encore le cas au début du chapitre III, 7. Montaigne y tient un discours sur l'ambition et déclare : «Et tout à l'opposite de l'autre, m'aimerais mieux deuxième ou troisième à Périgueux que premier à Paris $»^{64}$. Du livre III a disparu la figure du chef militaire dont on se remémore les glorieux combats, tout comme s'est évanoui l'historien que saluaient en particulier le chapitre II, $10^{65}$, et le chapitre II, 17, qui

${ }^{53}$ I, 47, VS. 282/P. 458 ; I, 48, VS. 288/P. 470 : César et Pompée, bons cavaliers ; I, 51, VS 305/P. 496 : «Pompeius, Cæsar, etc ... ont pris de là, leur grand appui à se monter à cette grandeur d'autorité ».

${ }_{54}$ «Si les gestes de Xenophon et de Cæsar», VS. I, 40, 249/ P. I, 39, 386 ; « Cæsar et Xenophon ont eu de quoi fonder et fermir leur narration», II, 18,VS. 663/P. 1024.

${ }_{55}$ I, 14, VS. 61/P. I, 40, 410 ; I, 48, VS. 288/ P. 470 « Comme nature a voulu faire de ce personnage et d'Alexandre deux miracles en l'art militaire »; II, 8, VS. 402/P. 637 « . ou qu'Alexandre et Cæsar aient jamais souhaité d'être privés de leurs glorieux faits de guerre...»;

${ }^{56}$ Nous soulignons.

${ }^{57}$ II, 11, VS. 424/ P. 670.

${ }^{58}$ VS. 1084/P. 1689.

${ }^{59}$ VS. 1073-1074/P. 1671.

${ }^{60}$ «Je ne sais si les exploits de Cæsar et d'Alexandre surpassent en rudesse la résolution d'une belle jeune femme », III, 5, VS. 861/P. 1348 ; III, 12, VS. 1058/P. $1644:$ «Et je trouve que Cyrus, Alexandre, Cæsar, ces trois maîtres du monde... »; III, 13, VS. 1108/P. 1727. «Quand je vois et Cæsar et Alexandre au plus épais de sa grande besogne...»

${ }^{61}$ III, 9, VS. 994/P. 1549 ; la dispute entre César et Pompée, III, 10, VS. 1014/P. 1576.

${ }^{62}$ Pharsale, VII, 320.

${ }^{63}$ VS. 802/ P. 1253. Nous soulignons.

${ }^{64}$ VS. 916B/ P. 1435. Peut-on lire ici un écho de ce que disait Dion Cassius : «Le désir de Pompée était de n'être le second de personne alors que celui de César était d'être le premier de tous » (XLI, 54, 1) ?

${ }^{65}$ VS. 416A/ P. 659. 
jugeait le style de César plus grand et moins aisé à imiter que celui de Salluste, et le rangeait aux côtés de Plutarque, plus inimitable que Sénèque ${ }^{66}$.

On pourrait déduire de ce changement de traitement que le jugement sur César a évolué, que l'admiration de Montaigne s'est émoussée. Désormais, comme l'a observé Catherine Séguier-Leblanc, les évocations des guerres civiles contemporaines accompagnent les mentions de Jules César en II, $33^{67}$ et II, $34^{68}$. L'aggravation de la situation en France ressemble de plus en plus à l'état de Rome entre César et Auguste. La seconde partie du chapitre II, 34 reprend le parallèle que Plutarque avait entrepris entre César et Alexandre et qu'il n'avait pas approfondi dans une comparaison. Montaigne pèse les qualités de chacun d'eux, et estime César «plus retenu et considéré dans ses entreprises $»^{69}$. Le parallélisme se poursuit deux chapitres plus loin («Des plus excellents hommes», II, 36), mais pour privilégier cette fois la figure d'Alexandre, en fustigeant l'ambition de César qui a «rencontré ce vilain sujet de la ruine de son pays $»^{70}$. De cette progression du chapitre 34 au chapitre 36, on pourrait conclure à une révision du jugement que Montaigne porte sur Jules César. Deux arguments textuels accréditeraient encore cette idée. Le premier concerne le chef militaire capable, comme Alexandre, de dormir au milieu des dangers ou de jouir des plaisirs de la vie. Dans le dernier chapitre des Essais, Montaigne met à égalité César et Alexandre pour louer les deux meneurs d'hommes :

Quand je vois et César et Alexandre au plus épais de sa sage besogne jouir si pleinement des plaisirs [...], je ne dis pas que ce soit relâcher son énergie, je dis que c'est la roidir ${ }^{71}$.

Deux additions (appartenant à la couche $\mathrm{C}$ du texte) viennent cependant infléchir l'éloge: la première qualifie, en incise, les plaisirs «naturels et par conséquent nécessaires et justes ». Mais elle ne renforce pas l'éloge de César comme d'Alexandre car elle est commentée par un second ajout qui conclut : «Sages s'ils eussent cru que c'était là leur ordinaire vacation, cette-ci l'extraordinaire». Autrement dit, César comme Alexandre sont dans l'erreur : capables d'apprécier les plaisirs simples et immédiats que procure la satisfaction des besoins élémentaires, ils leur ont pourtant préféré la gloire.

Le second exemple concerne plutôt César historien. Montaigne s'émerveille de voir « combien Cæsar se déploie largement à nous faire entendre ses inventions à bâtir ponts $»^{72}$. Mais en se relisant après 1588 , Montaigne commente ce passage pour

\footnotetext{
${ }^{66}$ VS. 638A/ P. 985.

${ }^{67}$ «Voilà des traits bien hasardeux selon mon jugement : et n'est pas merveille si aux guerres civiles, que nous sentons, ceux qui combattent, comme lui, l'état ancien de leur pays, n'en imitent l'exemple », II, 33, VS. 732A/P. 1139.

${ }^{68}$ VS. 742A/P. 1155. «Entre 1578 et 1588, le lecteur de César est devenu plus sévère pour le César de la guerre civile», dit C. Séguier-Leblanc, art.cité, p. 180.

${ }^{69}$ II, 34, VS. 739/ P. 1150.

${ }^{70}$ VS. 755 / P.1176.

${ }^{71}$ III, 13, VS. 1108B/ P. 1727.

${ }^{72}$ VS. I, 17, 72/P. I, 16, 113. Il reprend ce même exemple en II, 34: «Ce fut là qu'il bâtit ce pont admirable, de quoi il déchiffre particulièrement la fabrique : car il ne s'arrête si volon-
} 
regretter que César se soit étendu sur un sujet d'architecture dans lequel il n'avait aucune compétence, tandis qu'il «va se serrant, où il parle des offices de sa profession». Le jugement de fin de lecture que porte Montaigne sur son exemplaire des Commentaires de César va dans le même sens :

S'il dérobe rien à la verité j'estime que ce soit parlant de soi car si grandes choses ne peuvent pas être faites par lui qu'il n'y ait plus du sien qu'il n'y en met ${ }^{73}$.

Une autre phrase des Essais établit cette fois une dichotomie entre le faire et le dire qui vient contredire l'idée que chez César, l'un n'allait pas sans l'autre. Il s'agit d'une phrase du chapitre III, 12 :

il est bien plus aisé de parler comme Aristote et vivre comme Cæsar, qu'il n'est aisé de parler et vivre comme Socrates. Là loge l'extrême degré de perfection et de difficulté $^{74}$.

Voilà autant d'arguments qui pourraient faire croire que Montaigne s'est progressivement affranchi de la fascination qu'il éprouvait pour César. Pourtant, quand on regarde de près toutes les phrases des Essais consacrées à César, on prend conscience que les choses sont peut-être moins simples qu'elles ne paraissent et que chez Montaigne l'admiration pour César coexiste avec le refus de l'ambitieux.

\section{La coexistence et l'ambiguïté}

Cette coexistence de sentiments contradictoires suscités par Jules César se laisse particulièrement bien observer dans le chapitre II, 33, «Histoire de Spurina». Montaigne se demande si les passions de l'âme comme l'ambition ne sont pas plus difficiles à vaincre que les passions du corps (l'amour), et il s'attarde longuement sur l'exemple de Jules César dont il énumère les conquêtes féminines : «jamais homme ne fut plus adonné aux plaisirs amoureux $»^{75}$. Il ajoute à son portrait la suffisance en savoir et les talents d'orateur qui font la grandeur du personnage. Puis il s'attache aux «semences de vertu » qu'il avait en lui et dont témoignent sa sobriété (que Caton même est contraint de reconnaître ${ }^{76}$ ), mais aussi sa douceur et sa clémence envers les ennemis mêmes ${ }^{77}$ (il donne l'exemple de Caïus Memmius, de Caïus Cævus). Il souligne enfin la grandeur de son courage. Mais, à plusieurs reprises, il dit déplorer que toutes «ces belles inclinations » fussent «altérées par cette furieuse passion ambitieuse qui l'habitait» et lui fit faire de «cette grande République romaine» «un nom sans forme et sans corps». Autrement dit «ce seul vice» de

tiers en nul endroit de ses faits, qu'à nous représenter la subtilité de ses inventions, en telle sorte d'ouvrages de main», VS. 738/P. 1147.

${ }^{73}$ Somme, c'est Cesar, reprod. en fac-similé de l'exemplaire des Commentaires de César annoté par Montaigne, publié par A. Gallet, musée Condé, éd. W. Blake, 2002, page finale.

${ }^{74}$ VS. $1055 /$ P. 1639.

${ }^{75}$ VS. $729-730 / P .1134$

${ }^{76}$ VS. 731/P. 1137.

${ }^{77}$ Voir déjà VS. I, 24, 131/P. I, 23, 201 «il essaya par clémence et douceur à se faire aimer de ses ennemis mêmes ». 
l'ambition «perdit en lui le plus beau et le plus riche naturel qui fut onques ${ }^{78}$. Bien que le discours de Montaigne lui accorde un moins long développement qu'à la passion amoureuse, l'ambition est lourdement condamnée chez César.

Un jugement rapide en déduirait une évolution de l'admiration vouée à César. Mais ce serait compter pour rien le chapitre II, 34, qui suit l' "Histoire de Spurina» et célèbre (on l'a vu) le chef militaire sans plus mentionner la «pestilente ambition». La confrontation des deux passions dans le chapitre II, 33, et la succession même des deux chapitres traduit plutôt une coexistence chez Montaigne de sentiments partagés à l'égard de César.

Le même Montaigne qui souligne à plusieurs reprises la clémence de César et sa douceur envers les ennemis - douceur que La Boétie qualifie de $"$ venimeuse $»^{79}$ cite l'ordre que César donna à ses soldats «de tirer principalement au visage des gendarmes de Pompeius en la bataille de Pharsale» (au chapitre II, 27, "Couardise mère de cruauté $»)^{80}$. D'autre part, alors qu'au chapitre «De la Vanité », Montaigne déclare : «entre Cesar et Pompée, je me fusse franchement déclaré ${ }^{81}$, il rétablit en quelque sorte une forme d'égalité entre eux en estimant au chapitre suivant que leur discorde était simple «jalousie d'honneur et de commandement ${ }^{82}$ dénuée de malignité. Au chapitre «De la Colère », il donne un exemple d'animosité et d'âpreté de César ${ }^{83}$, qu'il commente de façon surprenante : «Le dire est autre chose que le faire, il faut considérer le prêche à part, et le prêcheur à part » ${ }^{84}$. Et trois chapitres plus loin, Montaigne fera l'éloge du chef militaire.

Il arrive encore que le même chapitre nous affronte à des vues sur César qui apparaissent contradictoires à ceux qui refusent le discernement et le distinguo. C'est le cas au chapitre II, 11. Montaigne évoque le suicide de Caton et déclare :

Je tomberais aisément en cette opinion, qu'il savait bon gré à la fortune d'avoir mis sa vertu à une si rude épreuve, et d'avoir favorisé ce brigand à fouler aux pieds l'ancienne liberté de sa patrie ${ }^{85}$.

Et quelques pages plus loin dans le même chapitre, dans la même couche de texte, il propose deux exemples ${ }^{86}$ qui témoignent de la clémence de César, «doux en ses vengeances $»^{87}$. Autrement dit, tout se passe comme si Montaigne se sentait tenu de

${ }^{78}$ VS. 733/P. 1140-1141.

${ }^{79}$ Discours de la servitude volontaire, éd. Vrin, 1947, p. 61.

${ }^{80}$ VS. 698C/P. 1080.

${ }^{81}$ III, 9, VS. 994B/P. 1549.

${ }^{82}$ III, 10, VS. 1014B/P. 1576.

${ }^{83}$ "Suetone récite, que Caïus Rabirius, ayant été condamné par Cæsar, ce qui lui servit le plus envers le peuple (auquel il appela) pour lui faire gagner sa cause, ce fut l'animosité et l'âpreté que Cæsar avait apporté en ce jugement. »

${ }^{84}$ II, 31, VS.715A/ P. 1110.

${ }^{85}$ VS. 424A/P. 670

${ }^{86}$ VS. 430/P. 680.

${ }^{87}$ Même contradiction au chapitre I, 49. Au titre des coutumes anciennes : Montaigne cite une parole de César indiquant une façon de combattre «sinistris sagos inuoluunt, gladiosque distringunt », VS. 297/P. 484. Puis, trois pages plus loin, il mentionne le surnom infamant qui fut attribué à César, qualifié de « ruelle du roi Nicomède », VS. 300/ P. 488. 
faire bonne mesure et d'équilibrer tout reproche formulé contre César par un éloge, ou inversement.

La succession des propositions elles-mêmes révèle encore ce qu'on hésite à appeler l'indécision ou la perplexité de Montaigne en face de César. Au chapitre I, 14 , il évoque les sacrifices consentis par qui «s'endetta d'un million d'or pour devenir César $»^{88}$. Cet énoncé était précédé d'une phrase qui justifiait, ou du moins comprenait un tel sacrifice : «Combien d'honnêtes hommes ont rejeté tout leur certain à l'abandon... pour chercher le vent de la faveur ». Mais la phrase concernant César est suivie d'une autre qui témoigne d'une légère inflexion due au changement de point de vue et de comparant puisque des honnêtes hommes on passe aux marchands : «Et combien de marchands commencent leur trafic par la vente de leur métairie ». Se trouvant ainsi situé dans une situation intermédiaire entre les honnêtes gens et les marchands, Jules César appartient à un entredeux indécidable. Un autre chapitre, le chapitre I, 47, offre encore un exemple d'énoncé ambigu. Montaigne est revenu sur la lutte entre César et Pompée et l'acharnement de César qui n'a pas su «mettre fin à sa convoitise». Il évoque ensuite les soldats que César faisait parer pour les inciter à combattre avec plus d'ardeur :

C'est toujours un aiguillon d'honneur et de gloire au soldat de se voir paré, et une occasion de se rendre plus obstiné au combat, ayant à sauver ses armes comme ses biens et héritages ${ }^{89}$.

Une réflexion de Xénophon s'ajoute à ce texte, puis l'énoncé reprend dans un sens tout différent :

Mais il s'offrirait aussi, de l'autre part, qu'on doit plutôt ôter au soldat le soin de se conserver, que de le lui accroître ; qu'il craindra par ce moyen doublement de se hasarder : joint que c'est augmenter à l'ennemi l'envie de la victoire par ces riches dépouilles.

On observe encore qu'à la fin du chapitre II, 34, qui louait l'art militaire de César, le général en chef a disparu et que Montaigne ne fait plus l'éloge que de ses soldats. Il serait hâtif d'en conclure à une démystification du héros, mais faut-il en inférer qu'il cherche à diminuer l'action de César, ou au contraire à l'exalter en montrant ce dont sont capables de simples soldats bien menés ? Il semble également que Montaigne hésite à attribuer à César ou à la fortune bien des vertus qu'il reconnaît au personnage historique. Les annotations aux Commentaires font apparaître combien il est sensible à la responsabilité que César sait accorder au hasard ${ }^{90}$. César lui apparaît à certains moments comme un miracle de la nature :

\footnotetext{
${ }^{88}$ VS. 63/P. I, 40, 413.

${ }^{89}$ VS. 283/P. 460.

${ }^{90}$ C'est par exemple le cas pour la Guerre Civile, livre III (p. 332 [452]), César écrivait : «Sed Casar, confisus fama rerum gestarum, infirmis auxiliis proficisci non dubitaverat, atque omnem sibi locum tutum fore existimabat», ce que Montaigne annote «Cesar se fioit en sa bonne fortune plus qu'au nombre de ses hommes ».
} 
comme nature a voulu faire de ce personnage et d'Alexandre deux miracles en l'art militaire, vous diriez qu'elle s'est efforcée à les armer admirablement. ${ }^{91}$

Mais il hésite ensuite à déterminer si ce qui a prévalu chez César est un volontarisme qui lui a fait renverser tous les obstacles par une absolue confiance en soi, ou une confiance en sa fortune. On constate que plus d'une fois dans les Essais se trouve soulignée cette confiance de César en la fortune :

À ses légions mutinées et armées contre lui, Cæsar opposait seulement l'autorité de son visage, et la fierté de ses paroles; et se fiait tant à soi et à sa fortune, qu'il ne craignait point de l'abandonner et commettre à une armée séditieuse et rebelle ${ }^{92}$.

Peut-être Montaigne suit-il ici Lucain qui faisait de César un personnage très confiant en sa fortune ${ }^{93}$. Mais il tente aussi d'expliquer «cette forte assurance»par l'idée que «l'imagination de la mort» n'effrayait pas César ${ }^{94}$. Cette fortune, c'est en quelque sorte la bonne étoile de César, que Montaigne associe étroitement à ses qualités personnelles :

Ce sont moyens extraordinaires, et qu'il n'appartient qu'à la fortune de Cæsar, et à son admirable prévoyance ${ }^{95}$.

On retrouve ce sens au chapitre 34 évoquant César qui « courut soudainement où la fortune le porta, pour exhorter ses gens $»^{96}$. Autrement dit, tant que César emploie ses efforts pour aller dans le sens même de la fortune, il a droit à l'indulgence, voire à l'admiration de Montaigne. Mais les choses changent si par son action, César contredit la fortune :

Il avait accoutumé de dire, qu'il aimait mieux la victoire qui se conduisait par conseil que par force. Et en la guerre contre Petreius et Afranius, la fortune lui présentant une bien apparente occasion d'avantage ; il la refusa, dit-il, espérant avec un peu plus de longueur, mais moins de hasard, venir à bout de ses ennemis ${ }^{97}$.

Voilà ce que Montaigne réprouve chez César: il lui semble faire preuve d'arrogance lorsqu'il veut gagner par soi, non grâce à l'aide du hasard. Peut-être

\footnotetext{
${ }^{91}$ I, 48, VS. 288/P. 470. En II, 33, il parle de son riche naturel, VS. 733 / P. 1140 : « Somme, ce seul vice, à mon avis, perdit en lui le plus beau, et le plus riche naturel qui fut onc » ${ }^{92}$ VS. I, 24, 130 /P. I, 23, 199.

${ }_{93}^{4}$ «Ici, c'est toi, Fortune, que je suis », au passage du Rubicon, Pharsale, I, 225-227.

${ }^{94}$ Le chapitre 33 parle de «résolution en la fortune contraire» (VS. 733/P. 1140), et dans le chapitre 34, Montaigne s'émerveille encore, tout en ramenant César à une catégorie plus générale : "Ces gens-là ont eu je ne sais quelle plus qu'humaine confiance de leur fortune » (VS. 740/P.1151).

${ }_{95}$ II, 33,VS. 733/P. 1139. Voir encore Lucain : «César aime le coup du destin qui va faire sombrer l'un ou l'autre », Pharsale, VI, 8-9; ou encore «de peur qu'il ne fût permis au destin de changer », II, 650 .

${ }^{96}$ VS. 738/P. 1147.

${ }^{97}$ VS. 739/P. 1149.
} 
touche-t-on là du doigt ce que Montaigne reproche le plus à César : partant de l'idée que la nature et le hasard prévalent sur l'art, il regrette que la vie et la réussite de César soient surtout l'effet de l'art. Quand, au chapitre II, 36, «Des plus excellents hommes », Montaigne compare César et Alexandre, on retrouve cette idée de fortune, différemment alléguée chez Plutarque ${ }^{98}$ :

Et il ne se peut nier, qu'il n'y ait plus du sien en ses exploits [il s'agit ici de César], plus de la fortune en ceux d'Alexandre.

Comme plus tard à Simon Goulart, très marqué par la lecture que les Essais ont faite d'Alexandre et de Jules César, César apparaît à Montaigne comme un parvenu de la politique ${ }^{99}$, qui n'a pas eu pour gouverner la même légitimité qu'Alexandre. Fils de roi, celui-ci a été poussé «par la vertu seule à commencer une guerre digne d'un grand Roy ${ }^{100}$. En face d'Alexandre qui a emprunté «le chemin royal de la vertu », César a été «tiré par son naturel et les tyranniques mœurs de son temps ${ }^{101}$. C'est déjà ce que disait Montaigne quand il cherchait à établir la responsabilité politique de César : «Quand l'ambition de Cæsar aurait de soi plus de modération, elle a tant de malheur, ayant rencontré ce vilain sujet de la ruine de son pays. $»^{102}$

\section{La pensée politique sur César: des Essais au Discours de la servitude volontaire}

On peut trouver quelque chose d'agaçant à l'association inévitable de Montaigne et La Boétie, et éprouver l'envie de disjoindre ces deux noms, de mettre de la zizanie dans ce couple - dont se gausse sans méchanceté une chanson de Georges Brassens ${ }^{103}$. Mais il serait étrange d'évoquer la figure de César dans la Renaissance française en faisant l'impasse sur le petit texte éblouissant qu'est le Discours de la servitude volontaire ${ }^{104}$ d'Étienne de La Boétie. Par un paradoxe apparent, La Boétie ne mentionne César que deux fois sous non nom. Mais s'agissant de la servitude

\footnotetext{
${ }^{98}$ Plutarque louait Alexandre d'avoir su affronter une fortune contraire qui écrivait «nul n'eut la fortune plus rebourse ni plus adversaire» qu'Alexandre, De la fortune ou vertu d'Alexandre, $\mathrm{II}^{\mathrm{e}}$ traité, III, 335B, $\mathrm{f}^{\circ}$ 316A.

${ }_{99}$ À noter : un homme de fortune désigne une personne qui s'est élevée rapidement. ${ }^{100} \mathrm{~F}^{\circ} 482^{\mathrm{v}} \mathrm{D}$.

${ }^{101}$ C'est ainsi qu'il « print à la malheure pour luy et pour son païs une teinture d'opiniastreté et d'ambition du tout insupportable, qui fut cause de sa mort», explique Simon Goulart dans sa Comparaison, op. cit., $\mathrm{f}^{\circ} 481^{\vee} \mathrm{E}$.

${ }^{102}$ II, 36, VS. 755 /P. 1176.

103 «Les Copains d'abord». Voir aussi et surtout: G. Mathieu-Castellani, «L'étrange vie posthume d'Étienne de La Boétie, ou les dangers de l'amitié », Étienne de La Boétie, sage révolutionnaire et poète périgourdin, éd. par M. Tetel, Paris, Champion, 2004, p. 333-348.

${ }^{104}$ É. de La Boétie, De la servitude volontaire ou contr'un, éd. par M. Smith, Droz, 1987 (abr. $D S V$ ). Comme pour tous les autres textes anciens, je cite dans la graphie modernisée, en respectant forme phonétique, ponctuation et majuscules de l'auteur.
} 
volontaire, s'agissant de cet «enchantement» mortel que le tyran exerce sur ses sujets ${ }^{105}$, comment le magistrat périgourdin n'aurait-il pas pensé à César ?

César, en réalité, est présent, tacitement ou secrètement, à tous les moments clés du raisonnement boétien, et des reflets fugaces de cette forte pensée se laissent deviner dans les Essais. En parcourant leur panorama des images de César, on peut, après avoir analysé, comme on vient de le faire, leur représentation d'une personnalité géniale, porter une lumière plus ciblée sur la question du politique, de la liberté et de la tyrannie. C'est alors que l'on est amené à deviner la présence de ce texte exhibé et caché, enfoui, et même peu à peu rejeté : le Discours de la servitude. Montaigne entretient avec cette pensée une relation ambiguë. La destinée de l'«ami per$\mathrm{du} »$ dans les Essais n'est pas ici notre objet ${ }^{106}$. En revanche on peut s'étonner de la disparition progressive des textes de La Boétie, le Discours d'abord, les «vingt et neuf sonnets » promis en remplacement ensuite, dans les chapitres- « cénotaphes » ${ }^{107}$ (comme le veut Michel Butor), I, 28 et 29, où pourtant a été maintenue l'annonce. Le cas de César permet de poser les questions qui touchent à ce problème. Montaigne hésite devant les deux textes politiques de La Boétie. Il ne mentionne que négligemment «quelques mémoires sur cet édit de Janvier» qui est aujourd'hui attribué de façon certaine à La Boétie ${ }^{108}$; quant au Discours de la servitude..., il le donne comme écrit «par manière d'essai en sa première jeunesse ${ }^{109}$, le définit lapidairement mais correctement «à l'honneur de la liberté contre les tyrans ", et l'apprécie sobrement comme "gentil et plein de ce qu'il est possible ${ }^{110}$. Les traces que La Boétie laisse dans les Essais sont intenses, affectives comme intellectuelles ${ }^{111}$, et ici on peut être étonné du manque d'enthousiasme dont témoignent ces lignes; à la fin de l'essai la suppression du texte du Discours est expliquée par des raisons politiques (l'usage que veulent en faire les séditieux), mais celle des poésies reste inexpliquée ${ }^{112}$. Plus étonnante encore la façon dont Montaigne définit ces deux textes politiques, pour nous essentiels, dans l'une des préfaces qu'il a données aux CEuvres de son ami, publiées chez Fédéric Morel en 1571: deux pièces dont il trouve «la façon trop délicate et mignarde pour les abandonner au grossier et pesant air d'une si

${ }^{105}$ «... un million d'hommes [...] enchantés et charmés par le nom seul d'un...» (DSV, p. 34).

${ }^{106}$ F. Charpentier, «Un ami que j' ai perdu », Montaigne Studies, La Boétie, vol. XI, 1-2, 1999, p. 197-208.

${ }^{107}$ Voir M. Butor, Essai sur les Essais, Gallimard, 1968.

${ }^{108}$ É. de La Boétie, Mémoire sur la pacification des troubles, éd. par M. Smith, Droz, 1983.

${ }^{109}$ Montaigne donne d'abord le Discours comme écrit par La Boétie à seize, puis dix-huit ans, soit en 1546 ou 1548 ; mais on s'accorde actuellement sur une date beaucoup plus tardive, vers 1554. Voir J. Balsamo, « [...] La Boétie et Machiavel», Montaigne Studies, vol. XI (cité n. 105), p. 10 ; et surtout G. Demerson, «Des exempla dans le $D S V$ : une rhétorique datée ?», Étienne de La Boétie, sage révolutionnaire et poète périgourdin, éd. M. Tetel, Champion, 2004, p. 195-224.

${ }^{110}$ Essais, I, 28 «De l'amitié », p. 183-184A. Je cite selon l'éd. Villey-Saulnier (VS), PUF, 1965, établie sur le texte de l'exemplaire de Bordeaux (EB); je donnerai également la page de l'éd. du Livre de Poche, sous la dir. de J. Céard (P), 2001, qui donne le texte procuré par Pierre de Brach et Marie de Gournay en 1595, lorsque celui-ci diffère de l'EB.

${ }_{111}$ À cet égard, voir tout le volume XI des Montaigne Studies cité n. 110.

${ }^{112}$ G. Defaux tente une explication séduisante, mais est-elle convaincante ? «Montaigne, La Boétie, les Essais », Montaigne Studies, XI, p. 169-198. 
malplaisante saison»; éditeur infidèle, il ne publie pas les deux traités. C'est pourquoi on a tenté ici une démarche «à rebours ", qui mène des Essais au Discours. On partira du César de Montaigne (si l'on peut ainsi l'appeler) pour constater qu'outre les sources bien connues et visibles, il se cache dans son livre un «César de La Boétie » palimpseste.

Les mentions de César, on l'a vu, sont nombreuses et réparties dans toute la période de rédaction des Essais. On constate cependant qu'elles se raréfient à mesure que le livre avance ${ }^{113}$; en tant que chef de guerre, César s'efface devant Alexandre dont les mentions au livre III remplacent en proportion celles de César aux deux premiers ; même lors de sa première rédaction de 1580 , dans son palmarès «des plus excellents hommes » (II, 36), moment où l'auteur s'apprête à refermer son œuvre, Alexandre l'emporte de justesse (je cite le texte de 1580) : «Ils ont eu plusieurs choses égales, et César à l'aventure aucunes plus grandes. Mais toutes pièces ramassées et mises en la balance, je ne puis que je ne penche du côté d'Alexandre » qui d'ailleurs est à son tour surpassé par «le tiers et le plus excellent [...], c'est Epaminondas $»^{114}$. Mais il est remarquable qu'à cet endroit, dans l'édition de 1588, Montaigne, comme pour éclairer son jugement, introduise la raison politique qui vaut à César son déclassement, par un paragraphe inséré entre les «[choses] plus grandes » accordées à César, et «toutes pièces ramassées » : «Ce furent deux feux ou torrents à ravager le monde [ici une citation de Virgile]. Mais quand l'ambition de César aurait de soi plus de modération, elle a tant de malheur, ayant rencontré ce vilain sujet de la ruine de son pays et de l'empirement universel du monde, que [toutes pièces ramassées, etc.] ». Quant à la personnalité de César, Montaigne a toujours été partagé entre l'admiration et la désapprobation, conforme en cela à ses sources : en matière de biographies, l'historiographie latine a soin de faire la part des vitia et des virtutes de chacun. En ce qui concerne les zones d'ombre de cette figure, Montaigne reste indulgent pour les plus regrettables, mais il va concentrer ses reproches sur l'aspect politique de l'action de César. On en trouve des échos dispersés jusque dans le dernier livre, mais exceptionnellement l'écrivain, qui ne consacre aucun autre chapitre à une monographie d'un grand homme ${ }^{115}$, construit de César, dès sa première édition, un tableau complet, dense, en deux volets, deux chapitres contigus vers la fin du livre II. Comme pour laisser la meilleure impression au lecteur, il place en second (II, 34, «Observations sur les moyens de faire la guerre de Julius Cæsar ») l'éloge, inconditionnel, du chef de guerre. Mais sous un titre trompeur (conformément à sa pratique humoristique des titre $\left.{ }^{116}\right)$, «L'histoire de Spurina», II, 33, dresse de César

\footnotetext{
113 «Avance» : entendons-le aux deux sens : avancée de l'auteur dans l'écriture, en observant les fréquences dans les «couches » A (1580), B (1588) et C (ajouts manuscrits de l'EB); et par ailleurs, avancée du lecteur dans la lecture, dans le «fil» du texte de la première vers la dernière page, du livre I au livre III, dans un ordre assurément voulu par Montaigne.

${ }^{114}$ Page 593 de l'édition de 1580 à Bordeaux, chez S. Millanges.

${ }^{115}$ On peut à la rigueur constater une exception au ch. 12 du livre III, «De la physionomie», qui est une sorte d'essai «De Socrate». « Du jeune Caton», I, 37, ne fait nullement un portrait de Caton et se trouve plutôt être une réflexion sur la poésie...

${ }^{116}$ Voir III, 9, «De la vanité »: «Les noms de mes chapitres n'en embrassent pas toujours la matière... », p. 994B.
} 
un portrait systématique. C'est en grande partie cet essai, puisque le trentequatrième a été largement présenté, que l'on va examiner.

L'obscur Spurina ${ }^{117}$ n'occupe qu'un paragraphe final de moins d'une dizaine de lignes en 1580. Dans l'Exemplaire de Bordeaux, Montaigne ajoutera à son essai un consistant «allongeail» qui généralise sa réflexion. Le chapitre a pris son point de départ dans les combats de passions contraires, dont le plus net est celui de l'amour et de l'ambition: il sera illustré par «l'exemple de Julius Cæsar»: quatre pages massives, interrompues seulement par une insertion (massive elle aussi) de l'EB sur «Mechmet, [...] qui subjuga Constantinople», et une ligne insérée dans l'éd. de 1588 (B), pour distinguer sa pratique amoureuse de celle de César. Quatre pages peu retouchées en somme; Montaigne, qui truffe si souvent son texte d'ajouts ou repentirs innombrables, ici n'a rien trouvé à changer, rien à nuancer ou ajouter à ce qu'il pensait en 1580 .

Dans «L'Histoire de Spurina» se lit une réflexion à la fois éthique et politi$q^{118}$. Ethique : elle décrit une personnalité contradictoire, subtile, nuancée ; exemplaire de la lutte en l'homme de passions contraires (une disparité d'appétits, comme dit Montaigne), et des plus fortes de toutes, l'amour et l'ambition ${ }^{119}$. Politique : elle concerne l'idéal de la république romaine, tenue pour le modèle de la liberté, mais d'une liberté utopique, ou du moins vite compromise: Montaigne se dira ailleurs «embabouiné » de Rome, mais, précise-t-il, «cette vieille Rome, libre, juste et florissante (car je n'en aime ni la naissance ni la vieillesse $\left.{ }^{120}\right) »$. Peut-être saisit-on dès à présent comme un reflet boétien dans la surface du texte montaignien: parlant de Caton (personnage sur lequel il faudra revenir), La Boétie prend soin de souligner : «on jugera qu'il était Romain et né dedans Rome, et lors qu'elle était libre» (p. $50)^{121}$. Lui non plus n'en aime ni les débuts (la royauté) ni la fin (l'empire). Pour ce chapitre 33, Montaigne ne recourt pas, comme source, à ses propres commentaires sur les Commentaires de César (bien qu'il soit largement nourri de leur lecture), et par exception, plutôt que «son» Plutarque (celui d'Amyot, les Vies traduites en 1559), il suit la Vie de C. J. César par Suétone ${ }^{122}$, comme le prouvent les détails qu'il a retenus, et sa démarche qui épouse à peu près le déroulement des chapitres de Suétone. Les premiers développements constituent la partie «éthique», cernant la personnalité de César. Ils mêlent assez étroitement l'admiration émue et des remarques qui frôlent le sarcasme. Comme il a amorcé son thème sur le parallèle (le combat) de l'amour et de l'ambition, il commence par montrer la «complexion

\footnotetext{
${ }^{117}$ Voir B. Boudou, «La mutilation de Spurina sous le scalpel de Montaigne (Essais, II, 33) », Littérales, Actes du colloque «Métamorphoses de la laideur», mai 2003, à Paris X-Nanterre, éd. par L. Picciola et C. Leroy, n 36, 2005, p. 89-104.

${ }^{118}$ Montaigne aime associer ces deux termes pour définir ce qu'est pour lui la «grande» histoire : «L'homme en général, que qui je cherche la connaissance, y paraît plus vif et plus entier...» (II, 10, 416A); il trouve dans «l'histoire de Tacitus » « une pépinière de discours éthiques et politiques » (III, 8, 941B).

${ }^{119}$ Parallèle qui passionnera l'âge classique, comme le montre le Discours sur les passions de l'amour autrefois attribué à Pascal, aujourd'hui rendu à Guilleragues.

${ }^{120}$ Essais, III, 9 « De la vanité », 996-997B.

${ }^{121}$ Il s'agit d'un passage où l'on voit Caton jeune tenté par un tyrannicide contre Sylla.

${ }^{122}$ Suétone, Vies des XII Césars, texte et trad. par H. Ailloud, Paris, Belles-Lettres, 19311932.
} 
amoureuse» de son héros: «jamais homme ne fut plus adonné aux plaisirs amoureux ». Montaigne n'insiste pas sur l'ambiguité sexuelle de César et, à part la mention de l'épisode de Bithynie avec le roi Nicomède ${ }^{123}$, il passe sous silence les ragots auxquels Suétone consacre plusieurs chapitres en rappelant pour finir le mot cruel : «le mari de toutes les femmes, la femme de tous les maris» (ch. 52); mais sa première mention touche «le soin curieux qu'il avait de sa personne [...], jusques à se servir à cela des moyens les plus lascifs qui fussent lors en usage, comme de se faire pinceter tout le corps et farder de parfums d'une extrême curiosité » $(729)^{124}$. La Boétie, dans son Discours, sans citer particulièrement César, insiste fortement sur la "paillardise» du tyran et son caractère efféminé, "non pas d'un Hercule ni d'un Samson, mais d'un seul hommeau, et le plus souvent le plus lâche et femmelin de la nation» (35). Cependant ces dispositions amoureuses ne sont pas ce qui suscite la réprobation de Montaigne ; avant même d'apporter un sérieux contre-point, de haute moralité celui-ci, à ces premiers traits, il accole au tableau des plaisirs son thème initial : celui de l'ambition :

Ses plaisirs ne lui firent jamais dérober une seule minute d'heure, ni détourner un pas des occasions qui se présentaient pour son agrandissement. Cette passion régenta en lui si souverainement toutes les autres, et posséda son âme d'une autorité si pleine, qu'elle l'emporta où elle voulut. Certes j'en suis dépit... (731).

À cette sévère remarque succèdent toutes les qualités qui forcent l'admiration chez César, énumérées avec émotion : qualités intellectuelles, sa science, son talent d'orateur, d'historien, d'écrivain ; et qualités morales, sa sobriété, sa simplicité, et surtout son courage, sa douceur et sa clémence, qu'il exerce en particulier dans la vie publique: "Jamais homme n'apporta ni plus de modération en sa victoire, ni plus de résolution en la fortune contraire» (733), conclut-il. Dans tout ce mouvement du texte, l'admiration semble l'emporter sur la critique et le portrait pourrait s'arrêter sur cette phrase. Mais Montaigne a laissé en attente le jalon de l'ambition, et c'est par ce «mais » que s'enchaîne la phrase suivante, sans alinéa dans les éditions anciennes (A comme B); cette passion devient folle, «furieuse » :

Mais toutes ces belles inclinations furent altérées et étouffées par cette furieuse passion ambitieuse, à laquelle il se laissa si fort emporter qu'elle tenait le timon et le gouvernail de toutes ses actions. D'un homme libéral elle en rendit un voleur public pour fournir à cette profusion et largesse... $(733)^{125}$

Tout ce passage frémit d'indignation. Les phrases en sont amples et martelées, largement subordonnées, marquées de termes vigoureux («voleur public, les plus méchants et perdus hommes du monde $» . .$.$) ; on peut admirer sa transcription du$ mot insupportable de César que rapporte Suétone - mais non Plutarque : «Il disait

\footnotetext{
${ }^{123}$ Voir aussi I, 49, «Des coutumes anciennes », où Montaigne cite Suétone : César, spondam regis Nicomedis, «ruelle du roi Nicomède » (Suétone, op. cit., ch. 49).

${ }^{124}$ Pour éviter une surcharge de notes, je donnerai, si possible, la page dans le corps du texte. Pour ce chapitre, toutes les citations sont de la couche A.

${ }^{125} 1580,1588$ et 1595 posthume, texte donné par l'éd. P, p. 1140.
} 
que la république était un nom sans réalité, sans valeur» (ch. 77); Montaigne : «Il osait se vanter en présence de ses concitoyens d'avoir rendu cette grande République Romaine un nom sans forme et sans corps» (733) scandé par les allitérations. Le portrait de César se clôt sur une condamnation sans appel. Tous ses traits admirables, exceptionnels, sont abolis dans la mémoire des hommes par ce «crime» que Montaigne lui reprochera encore au cours des Essais: «avoir voulu chercher sa gloire de la ruine de son pays et subversion de la plus puissante et fleurissante chose publique que le monde verra jamais » (733). La disposition typographique en un bloc massif, sans alinéa, contribue aussi à la force de ce texte. Sur quoi Montaigne abandonne l'exemple de César, pour se «remettre sur [ses] brisées », à savoir le nom de Spurina qui ornait le titre de l'essai. Le chef de guerre recevra un éloge sans réserve dans le chapitre suivant. Pourtant l'étoile montante d'Alexandre vient dans ce domaine le concurrencer. Plusieurs fois encore, César reviendra hanter les Essais. Ce n'est pourtant pas lui qui est choisi parmi les plus excellents pour mener l'œuvre vers sa fin en 1580, œuvre alors scellée, digne d'être offerte au roi. Comme l'a signalé $\mathrm{B}$. Boudou, moins présent après 1588 (dans le troisième livre), il est désormais accompagné d'un autre personnage (Alexandre, Pompée, ...). à qui on le compare. Il n'hésite pas, ressuscitant le contexte des guerres civiles, à opter pour le parti de Pompée, vaincu militaire de Pharsale : «Entre César et Pompée je me fusse franchement déclaré » (III, 9, 994B). En une occasion il l'appelle «l'autre» avec désinvolture (III, 7, 916B, à propos de «l'incommodité de la grandeur»!). Il n'apparaît qu'une seule mention manuscrite (EB) dans ce troisième livre ${ }^{126}$.

Ces remarques engagent la question de la pensée politique de Montaigne (qui ne peut évidemment être ici qu'effleurée) et de la présence de La Boétie dans les Essais. Par un paradoxe déjà souligné, César semble n'occuper qu'une place modeste dans le Discours de la servitude volontaire. La pensée politique de la Renaissance reconnaît généralement César comme un grand modèle tyrannique. L'historiographie latine transmet un portrait où l'accusation de tyrannie, sans être éludée, s'accompagne d'une reconnaissance de sa personnalité exceptionnelle. Cet équilibre du jugement est habituellement suivi par les historiens, les penseurs politiques : c'est ainsi que procède Montaigne. Or si César fait chez La Boétie une entrée subreptice, cette réserve est aussitôt contredite par le jugement impitoyable, sans appel, que l'auteur porte sur lui. Il a longuement déjà développé une théorie de la tyrannie où le personnage de César peut se deviner en filigrane, évoquant en particulier, parmi les grands héros libérateurs, «Brute et Casse » (contrairement à Montaigne, La Boétie francise les noms grecs et latins). On a pu déjà penser à César quand est évoquée la séduction du chef «femmelin » qui réussit à «enchanter» tant de sujets. C'est à propos de Néron (rapprochement éloquent) et à titre d'exemple qu'apparaît enfin le nom de César: La Boétie se scandalise des regrets qu'après sa mort a suscités « ce bourreau, cette bête sauvage », mais après tout, se dit-il, n'en a-til pas été de même

à la mort de Jules César, qui donna congé aux lois et à la liberté, auquel personnage il n'y eut, ce me semble, rien qui vaille (car son humanité même, que l'on prêche tant, fut plus dommageable que la cruauté du plus sauvage tyran qui fut oncques,

${ }^{126}$...et six dans les deux premiers. 
pource qu'à la vérité ce fut cette sienne venimeuse douceur qui envers le peuple romain sucra la servitude). (p. 59-60)

La Boétie se doit dans ce court traité (un «discours ») d'être dense. César est doublement caractérisé par son action et sa personnalité morale ; son action est envisagée dans sa finalité, essentiellement reconnue comme liberticide (donner congé aux lois et à la liberté); le jugement moral est lapidaire : "rien qui vaille », et les attendus en sont rejetés dans une parenthèse, qui prend le contre-pied de la doxa humaniste sur César. La Boétie ne croit ni à sa clémence ni à sa «douceur»; il fait l'hypothèse de l'hypocrisie dans toutes les conduites de César, notamment sa clémence tant vantée (que l'on prêche tant). Mais l'hypocrisie est une hypothèse insuffisante : la tyrannie est un poison mortel, auxquels les hommes se sont accoutumés par une sorte de mithridatisation, «comme l'on dit de Mithridate que se fit ordinaire à boire le poison, pour nous apprendre à ne trouver point amer le venin de la servitude» (p. 47). «En toutes contrées, en tout air est amère la sujétion» (p. 50). C'est pourquoi La Boétie parle de la «venimeuse» douceur de celui qui a «sucré » la servitude, la couvrant comme une pilule d'un enrobage trompeur.

En divers autres lieux des Essais on peut sentir cet affleurement des mots de La Boétie sous ceux de Montaigne, sur un certain nombre de réflexions de base. Outre la vision sans doute utopique de l'ancienne, la vertueuse république romaine $^{127}$, la conviction que l'emprise de César s'étendait sur le monde entier: Brutus et Cassius entreprenaient «la délivrance de Rome, ou plutôt de tout le monde » $(D S V$, p. 53), l'ambition de César allait à «l'empirement universel du monde » (Essais, II, 36, 755B) ; l'ambition de César s'alimentait de la corruption et des deniers publics, "D'un homme libéral elle en rendit un voleur public pour fournir à cette profusion et largesse » (Essais, II, 33, 733A), «De là venait la crue du Sénat sous Jules ${ }^{128}$ (Discours, p. 67). Plus largement, autour de César est habituellement évoquée une constellation de personnages et d'événements: Caton, l'opposant de César de longue date ; Brutus et Cassius, leur entreprise, leur erreur. Caton est mis par La Boétie au rang des champions de la liberté ; il le montre, pendant la dictature de Sylla, tenté par le tyrannicide: «Voilà certes une parole appartenante à Caton. C'était un commencement de ce personnage digne de sa mort» (p. 50). Le suicide de Caton apparaît comme une protestation contre la fin annoncée des libertés romaines; si on lit Plutarque de près, il a aussi et surtout été décidé parce que César, son ennemi, s'approchait d'Utique et qu'il ne voulait pas tomber dans ses mains ${ }^{129}$. La mort de Caton hante les Essais. Montaigne comme La Boétie voit dans son suicide l'ultime révolte de la liberté. Ce qu'il manifeste dans cet acte n'est pas seulement, selon lui, l'impassibilité stoïcienne : «il sentit du plaisir en une si noble action», et son souci de la chose publique (comparable à celui de Montaigne lui-même), lui fait considérer comme une chance de voir mettre «sa vertu à si belle épreuve, et d'avoir favorisé ce brigand [César] à fouler aux pieds l'ancienne liberté de sa patrie » (II, 11, 424A). Autour du meurtre de César tournent sans relâche ces quelques événements : défaite de Pompée, rôle de Caton, enchaînement des guerres civiles qui suivirent. Brutus et

${ }^{127}$... qui rayonne encore pour Montesquieu dans L'Esprit des lois.

${ }^{128}$ Deuxième des deux seules mentions du nom de César dans le Discours.

${ }^{129}$ Plutarque, Vie de Caton d'Utique, ch. 87-88, trad. d'Amyot. 
Cassius apparaissent comme des héros malheureux : «Brute le jeune et Casse ôtèrent bien heureusement la servitude, mais en ramenant la liberté ils moururent, non pas misérablement [...], mais certes au grand dommage, perpétuel malheur, et entière ruine de la république, laquelle fut, comme il semble, enterrée avec eux » (DSV, p. 54). «... C'est d'un jugement forcé [que Sénèque] condamne la cause de ces généreux meurtriers de César» (II, 10, 413A) ; mais l'échec du complot marque aussi la fin de la liberté romaine: «comme il advint aux tueurs de César, qui jetèrent la chose publique à tel point qu'ils eurent à se repentir de s'en être mêlés » (III, 9, 958C). Ce dernier trait prend pour Montaigne valeur d'exemple. Pris dans les luttes de son temps, il n'a pas à évoquer la perte d'une liberté qui n'entrait pas en considération dans un régime monarchique, mais il a la hantise de la discorde civile. D'où cette inflexion de son jugement. César n'est pas pour autant réhabilité, mais la pensée politique de l'écrivain n'est plus alors dominée par le concept de liberté : sur elle pèse son constat de la dégradation de l'État, de la ruine du royaume, ainsi que l'horreur des violences civiles, et son aspiration à la réconciliation dans une conception tolérante, encore bien lointaine alors. L'idée de liberté prend dans les Essais des voies intimes, intellectuelles, liées à sa philosophie du moi, tout autres que la liberté héroïque, prise dans une définition purement politique et romaine qu'il partageait avec La Boétie. La liberté reste toujours une valeur essentielle, il se dit plus que jamais «affadi » de liberté ${ }^{130}$, mais c'est dans l'«interne juridiction» qu'il entend exercer sur lui-même qu'il la trouve : «J'ai mes lois et ma cour pour juger de moi » (III, 2, 807B). Cet infléchissement est dû à l'importance croissante accordée à la sphère privée. Point de vue qui s'accorde à l'intériorisation progressive du jugement et des valeurs. Sa notion de la «grandeur» accompagne ce déplacement moral. Les grands modèles héroïques s'effacent devant des vertus moins éclatantes. Cette évolution s'annonçait très précocement: on a vu que la grandeur militaire d'Alexandre doit céder devant celle, plus humaine au cœur des batailles, d'Epaminondas. Caton lui-même, si admirable, lui paraît finalement toujours un peu «monté sur ses grands chevaux» (1038B). Lui aussi devra céder le pas devant le modèle suprême, Socrate. «Je conçois aisément Socrate en la place d'Alexandre; Alexandre en celle de Socrate, je ne puis » (809B). Alors, a fortiori, César...

La parole vivante de La Boétie produit sur Montaigne une impression profonde. Une vive exclamation traduit cette réception affective de la parole de l'ami : «Mais oyons un peu parler ce garçon de seize ans » $(\mathrm{I}, 28,194 \mathrm{~A})^{131}$. Sa vision idéale de Rome porte la marque de l'idéal républicain de La Boétie dans le Discours. César en est le contre-modèle absolu. Mais dans la galerie de portraits que la Renaissance offre de César, c'est La Boétie qui se démarque vigoureusement de la tradition humaniste. Montaigne, par les facettes multiples, par cet «éclatement» du portrait, veut-il apporter des retouches au César de La Boétie? Pour ma part je penserais plutôt que le modèle s'éloigne de lui, ou plutôt les modèles : celui de César, et aussi

\footnotetext{
${ }^{130}$ «Je suis si affadi après la liberté, que qui me défendrait l'accès de quelque coin des Indes, j'en vivrais aucunement [quelque peu] plus mal à mon aise »; "Toute ma petite prudence en ces guerres civiles où nous sommes, s'emploie à ce qu'elles n'interrompent ma liberté d'aller et venir », III, 13, 1072B.

${ }^{131}$ Sur la date réelle de ce texte et l'âge de son auteur au moment de sa rédaction, voir la note 108.
} 
le «visage» de l'ami, évoqué dans une remarque tardive et émouvante ${ }^{132}$, mais qui s'efface de plus en plus. Assurément César reste pour lui le grand chef de guerre, le grand écrivain, le grand historien qu'il a toujours été, mais il reste aussi le fossoyeur de la république romaine, et il rappelle trop tristement les «temps malades » que vit Montaigne. C'est désormais dans la conquête, ou plutôt la maintenance de sa souveraineté intime qu'il assure sa liberté.

Bénédicte Boudou Université de Paris-X-Nanterre

Françoise Charpentier Université de Paris VII-Denis Diderot

132 «Si à toutes forces je n'eusse maintenu un ami que j'ai perdu, on me l'eût déchiré en mille contraires visages », III, 9, 983B. 